Rabaska

Revue d'ethnologie de l'Amérique française

\title{
Entre la Bretagne et le Canada français : Donatien Laurent
}

\section{Jean-Pierre Pichette}

Volume 18, 2020

URI : https://id.erudit.org/iderudit/1072922ar

DOI : https://doi.org/10.7202/1072922ar

Aller au sommaire du numéro

Éditeur(s)

Société québécoise d'ethnologie

ISSN

1703-7433 (imprimé)

1916-7350 (numérique)

Découvrir la revue

Citer ce document

Pichette, J.-P. (2020). Entre la Bretagne et le Canada français : Donatien Laurent. Rabaska, 18, 298-302. https://doi.org/10.7202/1072922ar d'utilisation que vous pouvez consulter en ligne.

https://apropos.erudit.org/fr/usagers/politique-dutilisation/ 


\section{Entre la Bretagne et le Canada français : Donatien Laurent}

En août 2007, à Pointe-de-l'Église, Nouvelle-Écosse, l'Université SainteAnne accueillait un imposant colloque international ayant pour thème $L a$ Résistance des marges; sous-titré Exploration, transfert et revitalisation des traditions populaires des francophonies d'Europe et d'Amérique, il rassemblait trente et un chercheurs venus divulguer leurs travaux sur les migrations culturelles européennes vers l'Amérique. Scrutant des populations diverses observées du XVII ${ }^{\mathrm{e}}$ siècle à nos jours - tant les Français d'ici (Canadiens français ou Québécois de Charlevoix, Franco-Ontariens du Détroit, Métis de l'Ouest, Acadiens des Maritimes, de la Louisiane et de la Guyane), que de là-bas (Bretons de l'Hexagone ou de la Saskatchewan, Béarnais de la Californie, Wallons du Wisconsin ou même Burkinabés de France) -, leurs analyses dévoilèrent les visées des élites et les revirements des masses, signalèrent les controverses, les mises en scène et les ambiguïtés, ou encore relevèrent les transpositions, mutations et métissages opérés par ces marges dont la mémoire enracinée dans la durée marque l'identité, souvent à leur insu. Ces regards multipliés parachevaient en l'amplifiant une réflexion commune préalablement instillée par Donatien Laurent dans les marches laurentiennes.

\section{Des vues partagées}

L'idée germa en effet à Sudbury seize ans plus tôt. Déjà, quelques années auparavant, Marie-Louise Tenèze m'avait dirigé vers ce chercheur du CNRS pour éclairer le projet de recherche que je désirais entreprendre dans le prolongement de mes études doctorales : « c'est à Donatien Laurent, docteur ès lettres, chercheur au Centre d'ethnologie [de recherche] bretonne et celtique de l'Université de Brest, que je pense », et qui « passera cette année maitre de recherche », m'écrivait-elle en avril 1985. Après un long dialogue silencieux, resté épistolaire durant un intervalle laborieux qui m'éloigna du projet primitif, je rencontrais Donatien Laurent pour la première fois à l'automne 1991. Je l'avais tout spécialement convié à un colloque international pour saluer l'œuvre monumentale du jésuite Germain Lemieux, un folkloriste qui a voué sa vie à la recherche et à l'édition du patrimoine oral de l'Ontario 
français. Parmi les souvenirs inoubliables qu'évoque cette visite initiale, je retiendrai celui qui devait orienter la suite de mes travaux et de nos relations.

Invité à commenter la " place du conte populaire français d'Amérique dans la tradition orale de la francophonie », Donatien Laurent l'appréciait ainsi : « je ne suis pas éloigné de penser que dans ce sens [en termes de hiérarchie de qualité, de vitalité], et pour ce qui concerne son état de santé, il occupe la première place ». Justifiant sa position, il ajoutait que « les nombreuses publications faites ici depuis plus d'un demi-siècle [...] attestent de la façon la plus éclatante la vitalité des recherches menées au Canada français sur un terrain qui me parait, pour des raisons diverses, avoir beaucoup mieux résisté que sur le continent à la déstructuration du milieu traditionnel ${ }^{1} \gg$. S'interrogeant ensuite sur la façon dont cette « branche détachée du tronc continental » avait évolué en sol américain, il retenait que son expérience particulière « représente une sorte de laboratoire, de champ d'expérience et d'observation incomparable pour l'étude du conte populaire français de tradition orale et du fonctionnement de cette tradition », d'autant précieuse qu'elle « nous restitue un milieu qui chez nous, en France, a disparu presque partout depuis près d'un siècle : un véritable milieu de tradition orale ${ }^{2} »$. Notant en outre que l'Ontario français et la Bretagne « puise[nt] pour une large part [leurs] racines dans un terreau commun : 1'Ouest de la France, voire même la Haute-Bretagne », et reconnaissant que le caractère « celtique » du conte canadien " également présent en Basse-Bretagne [...] constitue lui aussi un trait d'union entre nos deux répertoires narratifs traditionnels qu'il conviendrait sans doute de mieux préciser », il terminait en souhaitant «que les relations nouées ici à l'occasion de ce colloque me permettent d'y contribuer $»^{3}$.

Ces propos inattendus coïncidaient exactement avec le bilan de mon étude comparative fraîchement publiée, notamment sur la continuité exemplaire de la tradition du conte populaire au Canada français. Comme la demi-douzaine de comparatistes de l'Université Laval l'avaient, à leur façon, montré avant moi $^{4}$, j'y soulignais, entre autres, que la tradition du Nouveau Monde n'avait, sur ce plan, rien à envier à celle de l'Ancien, ni pour le nombre des versions ni pour leur qualité, à un point tel que ma volumineuse recherche confirmait elle aussi « la vigueur de la tradition française transplantée au Canada, plus

1. D. Laurent, «La Place du conte populaire français d'Amérique dans la tradition orale de la francophonie ", dans Jean-Pierre Pichette (dir.), L'Euvre de Germain Lemieux, s.j. Bilan de l'ethnologie en Ontario français, Sudbury, Centre franco-ontarien de folklore et Prise de parole, «Ancrages », 1993, p. 276.

2. Ibid., p. 278.

3. Ibid., p. 284-285.

4. Dans des mémoires et thèses dirigés par Luc Lacourcière et publiés dans la collection « Les Archives de folklore » [«AF »] aux Presses de l’Université Laval [PuL] : Germain Lemieux, Hélène Bernier, Nancy Schmitz; ou restés inédits : Pauline Éthier, Margaret Low. 
riche et mieux conservée ici que dans son pays d'origine » tout en demeurant « très fidèle à ses sources européennes ${ }^{5}$ ». Donatien Laurent résumera cette conjonction dans sa lettre du 25 novembre 2003 : «J'ai pris connaissance de ce livre en 1991 [...]. En le parcourant, je me suis rendu compte à quel point ses conclusions se rapprochaient des remarques que j'y faisais sur le Canada français ».

Cette communion de pensée éclaire les rapports cordiaux qui s'établirent tout de suite entre nous et que nous avons maintenus au fil des ans. Mes projets s'orientaient alors vers les échanges interculturels pratiqués en Amérique du Nord dans certains domaines des traditions orales et sanctionnaient l'importance de l'analyse comparative que des études antérieures consacrées aux jurons et au conte populaire avaient privilégiées ${ }^{6}$.

\section{Un partenaire}

Le vœu de Donatien Laurent, formulé au terme de sa communication de Sudbury puis repris dans la dédicace de son ouvrage marquant, Aux sources du Barzaz-Breiz, qu'il offrit le mois suivant « à [s] on ami Jean-Pierre Pichette [...] en gage d'une collaboration que je souhaite longue et fructueuse entre nos deux Universités et leurs Centres de recherche sur la littérature orale ", ne resterait pas lettres mortes.

L'édition des actes du colloque Lemieux en 1993 eut bientôt des retombées notables quand Donatien m'invita à poursuivre ma réflexion, comme représentant du Canada français, dans le cadre du colloque international $\mathrm{La}$ Bretagne et la littérature orale en Europe qui commémorait à Quimperlé, en 1995, le centenaire de la mort de La Villemarqué ; l'exploration de la chanson traditionnelle, objet de mon propos, constata la même fascination des visiteurs et des chercheurs européens, français surtout, devant la haute qualité et la vitalité de la chanson folklorique française en Amérique alors que, selon leurs témoignages, elle s'était anémiée en Europe ${ }^{7}$. Par ailleurs, ce rassemblement fut pour moi une double révélation : d'abord de l'auteur du premier chansonnier breton qu'on célébrait enfin publiquement et dont les descendants présents étaient visiblement très fiers, mais surtout du formidable retentissement que la thèse de mon ami Donatien, à l'origine de

5. J.-P. Pichette, L'Observance des conseils du maître. Monographie internationale du conte type A.-T. 910 B précédée d'une introduction au cycle des Bons conseils (A.T. 910-915), Québec, PuL, «AF » 25 ; Helsinki, Academia Scientiarum Fennica, « Folklore Fellows Communications » 250, 1991, p. $570-571$.

6. J.-P. Pichette, Le Guide raisonné des jurons. Langue, littérature, histoire et dictionnaire des jurons, Montréal, Les Quinze, « Mémoires d'homme », 1980, 305 p.

7. J.-P. Pichette, « La Découverte de la chanson traditionnelle française en Canada ou la fascination d'un folklore vivifié », dans Fañch Postic (dir.), La Bretagne et la littérature orale en Europe, Mellac-Brest, Centre de recherche bretonne et celtique, Centre de recherche et de documentation sur la littérature orale, Centre international de rencontre des cultures de tradition orale, 1999, p. 261-289. 
cette réhabilitation, répercutait par toute la Bretagne. À son tour, mon collègue ajouterait une nouvelle pierre à cette recherche en concluant l'examen d'une chanson historique qu'il soumit pour les Mélanges Dupont : «Mais si l'on se bornait, pour en faire l'étude, à ne prendre en compte que les versions collectées en France, pays pourtant directement concerné par l'événement, on serait bien en peine. C'est l'apport des versions franco-américaines qui donne tout son poids et sa richesse à ce dossier ${ }^{8} »$.

Après cette lancée, la question du transfert des traditions orales européennes en Amérique du Nord, perceptible dans les échanges interculturels remarqués ici entre les groupes francophones, autochtones ou anglophones, allait teinter les projets de collaboration entre nous. Au cours d'une première mission à Brest, en mai 2000, je présentai à quelques chercheurs du Centre de recherche bretonne et celtique $(\mathrm{CRBC})$ l'ébauche du programme « Entre la France et le Canada français : la Bretagne » en vue de structurer diverses actions intéressant nos pays respectifs. La discussion se déplaça à Sudbury en octobre suivant quand Donatien vint donner une conférence publique sur «Un héritage du calendrier gaulois : le pèlerinage millénaire de la "troménie" de Locronan en Bretagne » et participer à quelques séminaires. Puis les interactions s'intensifieront. L'avènement de la revue d'ethnologie Rabaska, qu'il appuiera dès le départ en devenant membre du comité scientifique, lui fournira l'opportunité de publier un premier article dans le volume inaugural auprès de celui de son collègue du $\mathrm{CRBC}$, Jean-François $\operatorname{Simon}^{9}$; il récidivera dans le volume suivant avec le concours de Georges Delarue ${ }^{10}$.

Ces échanges régulièrement organisés, de part et d'autre de l'Atlantique, ont peu à peu fédéré une bonne douzaine de chercheurs d'Europe, regroupés autour du CRBC de l'Université de Bretagne occidentale, et autant d'Amérique, soutenus par la chaire de recherche COFRAm de l'Université Sainte-Anne et la Société québécoise d'ethnologie. Une trentaine d'événements, missions, congrès, rencontres et journées d'études les ont réunis tantôt à Sudbury, à Québec ou à Pointe-de-l'Église, tantôt à Brest, à Mellac ou à Quimperlé. Ainsi, Donatien et moi avons eu la bonne fortune de contribuer à ces nombreuses entreprises transatlantiques, auxquelles des collaborateurs formant réseau ont aussi apporté leur soutien ; parmi les plus assidus d'entre eux, se détachent

8. D. Laurent, « La Reddition du Foudroyant en 1758. Un épisode naval de la guerre de Sept Ans à travers la chanson française de tradition orale en France et en Nouvelle-France ", dans Entre Beauce et Acadie. Facettes d'un parcours ethnologique. Études offertes au professeur Jean-Claude Dupont. Textes réunis par J.-P. Pichette avec la collaboration de J. Mathieu, R. Dubé et Y. Bergeron, [Québec], PuL, [2001], p. 260.

9. D. Laurent, " "La Belle qui fait la morte". Emprunt et adaptation : les versions en langue bretonne », Rabaska, no 1, 2003, p. 21-29; Jean-François Simon, « La Hache, outil fondateur breton à Saint-Brieux, Saskatchewan? », ibid., p. 31-42.

10. D. Laurent et G. Delarue, «Points de vue : Répertoire des chansons françaises de tradition orale. Comparaison des catalogues Coirault et Laforte », Rabaska, vol. 2, 2004, p. 159-167. 
les noms de Fañch Postic et de Jean-François Simon chez nos partenaires de la Bretagne, de Jean Simard et de Ronald Labelle au Canada français.

\section{Un hommage}

Le samedi 18 août 2007, à la baie Sainte-Marie, Donatien réservait à la Résistance des marges sa dernière prestation en carrière ${ }^{11}$. Ce grand colloque, dont il avait inspiré la thématique et que nous avons, avec lui et surtout grâce à lui, concrétisé en une manifestation pluridisciplinaire d'envergure internationale, se mua en hommage au discret artisan qu'il avait été. Si ce jour marquait pour lui l'aboutissement personnel d'un vif intérêt pour la francophonie de ce pays, consolidé par les liens cordiaux tissés depuis les années 1960 avec les Lacourcière, Lemieux, Laforte ou Schmitz, puis renouvelés avec notre génération, son influence continuerait encore par ces participations «britto-franco-canadiennes » à d'autres projets conjoints qui ont conduit à de nouveaux colloques internationaux - sur le passage de l'oral à l'écrit (Pointe-de-l'Église, 2008), sur les prêtres collecteurs (Pointede-l'Église et Brest, 2011) et sur l'œuvre de Marius Barbeau (Saint-Irénée, 2014) - et à de constantes collaborations à la revue Rabaska.

À la fin d'une visite amicale au couple Laurent, à leur maison de la rue de Lorient, où nous avions plaisamment ressassé notre association longue de trois décennies, il me dédicaça son dernier-né, Parcours d'un ethnologue en Bretagne (Brest, Empleo Breiz, 2013), une anthologie de ses écrits épars : « À Jean-Pierre Pichette, mon "double" québécois, en souvenir de son passage à Brest, et de nos échanges passionnés... 18 octobre 2014, Donatien Laurent».

Mon collègue Fañch Postic me confiait récemment que, dans l'esprit de Donatien, « cela ne faisait pas l'ombre d'un doute que les francophonies du Canada devaient être associées », pour l'avancement des études en littérature orale, avec les chercheurs du CRBC qu'il dirigeait. C'est pourquoi il nous avait mis en relation au moment des colloques de Mellac et de Quimperlé en 1995. «Plusieurs fois, ajoute Fañch, Donatien m'a dit combien il était heureux qu'avec Jean-François Simon, j'aie continué à maintenir les liens qu'il avait contribué à nouer $»$.

Je garde le souvenir d'un chercheur patient, de ses intuitions géniales, qui avait donné un sens et encouragé des projets que j'envisageais isolément ; d'un homme de cœur et d'une belle simplicité, avec bien sûr son sourire moqueur et ses distractions épiques, mais qui m'avait honoré de son amitié.

JeAn-Pierre Pichette Université Sainte-Anne

11. D. Laurent, « Le Rôle des marges linguistiques dans la transmission des chansons de tradition orale - Quelques remarques sur les versions du "Roi Renaud" en Bretagne », op. cit., Port-Acadie, $\mathrm{n}^{\text {os }} 13-14-15,2008-2009$, p. 447-455. 